\title{
Impact of visit-to-visit variability of blood pressure on deterioration of renal function in patients with non-diabetic chronic kidney disease
}

\begin{abstract}
Kei Yokota ${ }^{1,2,3}$, Masamichi Fukuda ${ }^{2}$, Yoshio Matsui ${ }^{1,4}$, Satoshi Hoshide ${ }^{1}$, Kazuyuki Shimada ${ }^{1}$ and Kazuomi Kario ${ }^{1}$
An association between visit-to-visit variability (VVV) of blood pressure (BP) and renal damage was recently reported in a cross-sectional study. We aimed to clarify the longitudinal effect of VVV of BP on deterioration of renal function in patients with non-diabetic chronic kidney disease (CKD). We retrospectively studied 56 patients with non-diabetic CKD (stage 3 or 4) who visited our nephrology clinic between September 1994 and May 2011. VVV of BP was defined as the standard deviation and coefficient of variation (CV) of office BP measured at 12 consecutive visits. Main outcomes were the annual decline in the estimated glomerular filtration rate (eGFR) and the composite renal end point defined as a doubling of serum creatinine or the need for dialysis. The median observation period was 83 months. Standard deviation and CV of office systolic BP (SBP) were significantly associated with the slope of the eGFR after adjustments for confounders. The adjusted risk for composite renal end points more than doubled for each increment of 1-standard deviation of the standard deviation of office SBP (hazard ratio (HR) 2.20, $P=0.001$ ), and for each increment of 1-standard deviation of the CV of office SBP (HR 2.12, $P=0.002$ ). The present study demonstrated that the visit-to-visit variability of BP is an independent determinant of deterioration of renal function in patients with non-diabetic CKD.
\end{abstract}

Hypertension Research (2013) 36, 151-157; doi:10.1038/hr.2012.145; published online 27 September 2012

Keywords: blood pressure; chronic kidney failure; glomerular filtration rate

\section{INTRODUCTION}

Due to its increasing prevalence and incidence, chronic kidney disease (CKD) is a major public health concern worldwide. ${ }^{1-3}$ The demand for renal replacement therapy (dialysis and transplantation) is growing, and this growth has imposed a substantial economic burden on the medical care systems of many nations. The rate of renal function decline is reported to be associated with cardiovascular and all-cause mortality. ${ }^{4,5}$ Identification of rapidly progressive CKD and aggressive modification of risk factors are of paramount importance.

Hypertension is the most prevalent independent risk factor for the development of CKD. ${ }^{6-11}$ The prevalence of hypertension is as high as $80-85 \%$ in patients with CKD. ${ }^{12}$ Good control of the mean blood pressure (BP) level is a key component in the management of CKD. Guidelines from the Kidney Disease Outcomes Quality Initiative, the Seventh Report of the Joint National Committee (JNC 7), and the American Heart Association propose a BP treatment target goal of $<130 / 80 \mathrm{~mm} \mathrm{Hg}$ in patients with CKD. ${ }^{13-15}$

Recently, visit-to-visit variability (VVV) of BP has been found to be a strong risk factor for stroke and myocardial infarction, and for all-cause mortality independent of the mean office BP level. ${ }^{16-19}$ In a cross-sectional study, the VVV of BP was reported to be correlated with renal damage, as measured by albuminuria, and the resistive index, as evaluated by renal Doppler ultrasonography, which is thought to be a good indicator of renal vascular resistance. ${ }^{20}$ In addition, in a retrospective cohort study of patients with type 1 diabetes mellitus, it was demonstrated that the VVV of BP was associated with the development of nephropathy, defined as the development of albuminuria. ${ }^{21}$ However, there are still no data available regarding the longitudinal relationship between the VVV of BP and changes in renal function in patients with non-diabetic CKD.

We hypothesized that the VVV of BP might be associated with a deterioration of renal function independent of the mean BP level in patients with non-diabetic CKD. We investigated the impact of the $\mathrm{VVV}$ of BP on the annual rate of decline in renal function, estimated as the slope of the estimated glomerular filtration rate (eGFR). In addition, we also investigated the impact of the VVV of BP on composite renal end points, defined as either a doubling of serum creatinine or the need for dialysis. ${ }^{22}$

\footnotetext{
${ }^{1}$ Department of Medicine, Division of Cardiovascular Medicine, Jichi Medical University School of Medicine, Tochigi, Japan; ${ }^{2}$ Department of Nephrology, Iwakuni Medical Center, Yamaguchi, Japan; ${ }^{3}$ Department of Internal Medicine, Hongo Municipal Clinic, Yamaguchi, Japan and ${ }^{4}$ Department of Internal Medicine, Iwakuni Medical Center, Yamaguchi, Japan

Correspondence: Dr K Yokota, Department of Medicine, Division of Cardiovascular Medicine, Jichi Medical University School of Medicine, 3311-1 Yakushiji, Shimotsuke, Tochigi 329-0498, Japan.

E-mail: m02097ky@jichi.ac.jp

Received 7 May 2012; revised 10 July 2012; accepted 13 July 2012; published online 27 September 2012
} 


\section{METHODS}

\section{Study patients}

We retrospectively studied patients with CKD who visited our nephrology clinic at Iwakuni Medical Center from September 1994 to May $2011(n=393)$. Patients with diabetes mellitus were excluded $(n=105)$. Patients were excluded if they were pregnant $(n=5)$ or had severe systemic conditions such as an acute inflammatory state $(n=7)$, collagen disease $(n=25)$, cirrhosis $(n=23)$ and hematologic disease $(n=10)$. Patients with active renal diseases such as glomerulonephritis $(n=92)$, and congenital urological abnormalities such as unilateral kidney $(n=11)$, polycystic kidney disease $(n=26)$ and reflux nephropathy $(n=3)$ were also excluded. The clinical course of patients with CKD was followed through the medical charts until the start of dialysis, all-cause death, or May 2011, whichever was earliest. ${ }^{8}$ We enrolled patients with CKD stage 3 or $4\left(\text { eGFR } 15-59 \mathrm{ml} \mathrm{min}^{-1} \text { per } 1.73 \mathrm{~m}^{2}\right)^{23}$ at the first visit. In cases when patients were alive without the need for dialysis in May 2011, patients were excluded if their observation periods were $<4$ years. We ultimately enrolled 56 patients. This study was approved by the Institutional Review Board of Iwakuni Medical Center, and written informed consent was obtained from all participants.

\section{Study design}

The present study was a retrospective observational study. The patients' visits were separated by an interval of 1-12 months. At the beginning of the observation period, angiotensin-converting enzyme inhibitors or angiotensinreceptor blockers were prescribed to all patients for renal protection. ${ }^{14,24}$ Target BP was $130 / 85 \mathrm{~mm} \mathrm{Hg}$ before 2003 according to the fifth report of Joint National Committee on Detection, Evaluation, and Treatment of High Blood Pressure (JNC) 5 and JNC $6 ;^{25,26}$ and the target BP was $130 / 80 \mathrm{~mm} \mathrm{Hg}$ after 2003 , according to JNC $7 .{ }^{9}$ If the patients had not reached the target BP, then the other antihypertensive agents were added. Dietary protein was restricted to $0.8 \mathrm{~g} \mathrm{~kg}^{-1}$ per day. ${ }^{14,27}$ Dietary sodium intake was restricted to $5 \mathrm{~g}$ of sodium chloride per day. ${ }^{14}$ All patients underwent a medical interview, anthropometric measurements, and blood and urine examinations in the morning after having fasted overnight. Habitual drinking was defined as alcohol intake on $>5$ days per week, regardless of the amount. The threshold for dipstick proteinuria was set at $1 \mathrm{gl}^{-1} .28$

\section{BP measurements}

Office BP and heart rate were measured simultaneously by attending nurses in the morning with a mercury sphygmomanometer. At each office visit, two consecutive readings were taken on the non-dominant arm with a 1-min interval after 5 min rest in a sitting position; the average of two readings was adopted as the office BP and heart rate

\section{Definition of BP variability and BP instability}

Over a series of 12 consecutive visits from the beginning of the observation period, the mean office BP and the VVV of BP (expressed as within-individual standard deviation (s.d.) and coefficient of variation $(\mathrm{CV}) ; \mathrm{CV}=$ s.d./mean office $\mathrm{BP}$ in the 12 visits $\times 100(\%))$ were measured. The BP instability indices, expressed as the maximum office $\mathrm{BP}^{18}$ or the delta in office $\mathrm{BP}$ for the 12 visits, were also measured. Delta in BP was defined as a difference between the maximum and the minimum $\mathrm{BP}^{29}$

\section{Measurement of renal function \\ Serum creatinine was measured at each office visit using an enzymatic method (before 2005 March with the AutoAnalyzer 7150, Hitachi, Tokyo, Japan; after 2005 April with the AU 640, Beckman Coulter, Brea, CA, USA). Estimated GFR was calculated using the 4-variable Modification of Diet in Renal Disease (MDRD) equation with a Japanese coefficient of 0.808 calculated as follows: ${ }^{30}$ eGFR $\left(\mathrm{ml} \mathrm{min}^{-1}\right.$ per $\left.1.73 \mathrm{~m}^{2}\right)=0.808 \times 175 \times$ serum creatinine $^{-1.154} \times$ age $^{-0.203} \times 0.742$ (if female)}

\section{Definition of renal outcomes}

The primary outcome was the rate of decline in renal function, estimated by fitting a regression line through the eGFR measurements for each individual patient. $^{8}$ This resulted in slopes expressing the yearly decrease in eGFR. Estimated GFR measurements at the beginning and the end of the observation period were used. The secondary outcome was the composite renal end point, defined as a doubling of serum creatinine or the need for dialysis. A doubling of creatinine was defined as the first serum creatinine value that was twice the baseline value, confirmed by a similar second value at least 4 weeks after the finding of a doubling. ${ }^{31}$

\section{Statistical analyses}

All data are expressed as the mean values \pm s.d. or as a percentage, unless otherwise specified. Univariate correlations between the BP parameters and the slope of the eGFR were assessed using Pearson's correlations. After adjustments were made for possible confounding factors (model 1: age, sex, body mass index (BMI), habitual drinking, ${ }^{32}$ dipstick proteinuria $\left(\geqslant 1 \mathrm{gl}^{-1}\right.$ threshold), ${ }^{28}$ and mean heart rate, ${ }^{33}$ model 2: age, sex, BMI, habitual drinking, current smoking, ${ }^{34}$ statin use, ${ }^{35}$ fasting glucose, ${ }^{36}$ low-density lipoprotein cholesterol, ${ }^{37}$ dipstick proteinuria, mean heart rate and mean interval between visits), multivariate linear regression analyses on the slope of the eGFR were performed. The s.d. of office BP, CV of office BP, maximum office BP and delta in the office BP were also adjusted for mean office systolic blood pressure (SBP). The Kaplan-Meier method was used to construct cumulative time-toevent curves for two groups divided by the median CV of office SBP and by the median s.d. of office SBP. The comparisons between two groups were based on a log-rank test. Cox regression analyses with BP parameters were used to estimate hazard ratios (HRs) and 95\% confidence intervals (CIs) for composite renal end point, with or without adjustments for age, sex, BMI, habitual drinking, dipstick proteinuria and mean heart rate. Model assessment for the proportionality of hazard was based on plots of $\log (-\log$ (survival) $)$ vs. $\log$ (time). To identify cutoff values for VVV of BP as an indicator of the composite renal end point, receiver operating characteristic curves were constructed. The Youden index, an index that gave the same weight to false positive and false negative results, was used to determine the thresholds. ${ }^{38}$ The Youden index was defined as (sensitivity + specificity) -1 . Differences in the VVV of SBP or the slope of the eGFR according to antihypertensive medication use were assessed by unpaired $t$-tests between the two groups, subdivided by antihypertensive medication use. The null hypothesis was rejected when the two-tailed $P$-value was $<0.05$. All statistical analyses were performed with SPSS version 19 (SPSS, Chicago, IL, USA).

\section{RESULTS}

\section{Characteristics of the patients}

Characteristics of the study patients are shown in Table 1 . The mean age of the total patients was 69.5 years. At baseline, the mean serum creatinine was $137 \mu \mathrm{moll}^{-1}$, mean eGFR was $37 \mathrm{ml} \mathrm{min}^{-1}$ per $1.73 \mathrm{~m}^{2}$ and mean office BP was $140 / 78 \mathrm{~mm} \mathrm{Hg}$. The median observation period was 83 months (interquartile range 57-116). The median period of 12 consecutive visits from the beginning of the observation period was 24 months (interquartile range 13-38). The average of the mean interval between visits was 2.3 months. At the end of the observation period, the mean serum creatinine level was $296 \mu \mathrm{moll}^{-1}$ and the mean eGFR was $22.5 \mathrm{ml} \mathrm{min}^{-1}$ per $1.73 \mathrm{~m}^{2}$. The mean slope of the eGFR was $-2.03 \mathrm{ml} \mathrm{min}^{-1}$ per $1.73 \mathrm{~m}^{2}$ per year, which was approximately two times the rate previously described in studies of normal aging. ${ }^{39}$

\section{Univariate correlations of VVV of BP with the slope of the eGFR} The s.d. of office SBP/diastolic blood pressure (DBP) and the CV of office SBP/DBP were significantly correlated with the slope of the eGFR $(r=-0.50, P<0.001 / r=-0.28, P=0.036 ; r=-0.43$, $P=0.001 / r=-0.30, P=0.023$ ) (Table 2; Figure 1). Mean office SBP, maximum office SBP and delta in office SBP were also significantly correlated with the slope of the eGFR $(r=-0.29$, $P=0.028 ; r=-0.48, P<0.001 ; r=-0.51, P<0.001)$. Other clinical variables of $\mathrm{BP}$ parameters and blood biochemical examinations were 
Table 1 Characteristics of the patients

\begin{tabular}{|c|c|}
\hline & Non-diabetic CKD $(\mathrm{n}=56)$ \\
\hline Age, years & $69.5 \pm 11.8$ \\
\hline Male sex, \% & 59 \\
\hline $\mathrm{BMI}, \mathrm{kg} \mathrm{m}^{-2}$ & $23.5 \pm 4.1$ \\
\hline Current smoking, \% & 30 \\
\hline Habitual drinking, \% & 36 \\
\hline Hypertension, \% & 82 \\
\hline Dyslipidemia, \% & 54 \\
\hline Coronary artery disease, \% & 11 \\
\hline Stroke, \% & 9 \\
\hline \multicolumn{2}{|c|}{ Antihypertensive agents other than ACE inhibitor and $A R B$} \\
\hline Calcium channel blocker, \% & 50 \\
\hline Diuretic, \% & 30 \\
\hline Beta blocker, \% & 11 \\
\hline Alpha blocker, \% & 11 \\
\hline Statin, \% & 41 \\
\hline Serum creatinine, $\mu \mathrm{mol} \mathrm{I}^{-1}$ & $137 \pm 51$ \\
\hline eGFR, $\mathrm{ml} \mathrm{min}^{-1}$ per $1.73 \mathrm{~m}^{2}$ & $37 \pm 13$ \\
\hline BUN, mmoll-1 & $8.9 \pm 3.7$ \\
\hline Serum uric acid, $\mu \mathrm{mol} \mathrm{I}^{-1}$ & $395 \pm 116$ \\
\hline Hemoglobin concentration, $\mathrm{gl}^{-1}$ & $125 \pm 21$ \\
\hline Fasting glucose, $\mathrm{mmoll}^{-1}$ & $5.8 \pm 1.1$ \\
\hline Hemoglobin A1c, \% & $5.6 \pm 0.4$ \\
\hline Serum total cholesterol, mmoll-1 & $5.5 \pm 1.1$ \\
\hline Serum LDL cholesterol, mmol ${ }^{-1}$ & $3.3 \pm 0.9$ \\
\hline Serum HDL cholesterol, mmol I-1 & $1.6 \pm 0.7$ \\
\hline Serum triglyceride, $\mathrm{mmoll}^{-1}$ & $1.3 \pm 0.4$ \\
\hline Dipstick proteinuria $\geqslant 1 \mathrm{gl}^{-1}, \%$ & 18 \\
\hline Mean office heart rate, b.p.m. & $70 \pm 8$ \\
\hline Mean office SBP, mm Hg & $140 \pm 14$ \\
\hline Mean office DBP, mm Hg & $78 \pm 9$ \\
\hline The s.d. of office SBP, $\mathrm{mm} \mathrm{Hg}$ & $15.3 \pm 6.1$ \\
\hline The s.d. of office DBP, $\mathrm{mm} \mathrm{Hg}$ & $8.7 \pm 3.2$ \\
\hline CV of office SBP, \% & $10.9 \pm 4.2$ \\
\hline CV of office DBP, \% & $11.5 \pm 4.2$ \\
\hline Maximum office SBP, mm Hg & $166 \pm 21$ \\
\hline Maximum office DBP, $\mathrm{mm} \mathrm{Hg}$ & $93 \pm 11$ \\
\hline Delta in office SBP, $\mathrm{mm} \mathrm{Hg}$ & $51 \pm 22$ \\
\hline Delta in office DBP, $\mathrm{mm} \mathrm{Hg}$ & $29 \pm 11$ \\
\hline
\end{tabular}

Data are shown as the mean \pm s.d. or percentage.

Abbreviations: ACE, angiotensin converting enzyme; ARB, angiotensin receptor blocker; BMI, body mass index; b.p.m., beats per minute; BUN, blood urea nitrogen; CKD, chronic kidney disease; $\mathrm{CV}$, coefficient of variation; DBP, diastolic blood pressure; eGFR, estimated glomerula filtration rate; HDL, high-density lipoprotein; LDL, low-density lipoprotein; SBP, systolic blood pressure.

not significantly correlated with the slope of the eGFR, although blood urea nitrogen and hemoglobin concentration were marginally significantly correlated with the slope of the eGFR $(r=-0.25$, $P=0.06 ; r=0.26, P=0.06)$.

\section{Multivariate regression analyses between $\mathrm{VVV}$ of $\mathrm{BP}$ and the slope} of the eGFR

Table 3 shows the results of the multivariable linear regression analyses that determined the independent association of BP parameters with the slope of the eGFR after adjustments for confounders. We did not observe any multicolinearity in any models; all variance inflation factors were <3.0. In the model 1, the s.d. of office SBP/DBP, the CV of office SBP/DBP, the maximum office SBP
Table 2 Univariate correlations on the slope of the eGFR in patients with non-diabetic CKD $(n=56)$

\begin{tabular}{lcc}
\hline & $r$ & $P$ \\
\hline Mean office SBP, mm Hg & -0.29 & 0.028 \\
Mean office DBP, mm Hg & 0.06 & 0.64 \\
The s.d. of office SBP, mm Hg & -0.50 & $<0.001$ \\
The s.d. of office DBP, mm Hg & -0.28 & 0.036 \\
CV of office SBP, \% & -0.43 & 0.001 \\
CV of office DBP, \% & -0.30 & 0.023 \\
Maximum office SBP, mm Hg & -0.48 & $<0.001$ \\
Maximum office DBP, mm Hg & -0.02 & 0.91 \\
Delta in office SBP, mm Hg & -0.51 & $<0.001$ \\
Delta in office DBP, mm Hg & -0.23 & 0.09 \\
\hline
\end{tabular}

Abbreviations: CKD, chronic kidney disease; CV, coefficient of variation; DBP, diastolic blood pressure; eGFR, estimated glomerular filtration rate; SBP, systolic blood pressure.

and the delta in office SBP were independently associated with the slope of eGFR. In the model 2, the s.d. of office SBP, the CV of office SBP/DBP, the maximum office SBP and the delta in SBP were independently associated with the slope of the eGFR. On the other hand, the correlation between mean office SBP and the slope of the eGFR was not significant after adjustments for confounders in the both models.

Impact of VVV of BP on composite renal end points

To evaluate the relationship between VVV of BP and renal outcomes, we divided the patients with non-diabetic CKD into two groups according to the median s.d. of office SBP (low-s.d. group, $n=28$; high-s.d. subgroup, $n=28$ ) and the median CV of office SBP (low-CV group, $n=28$; high-CV subgroup, $n=28$ ). Figure 2 shows KaplanMeier curves for composite renal end point, that is, for the doubling of serum creatinine or the need for dialysis. Log-rank tests demonstrated that the risk of composite renal end points was associated with increasing s.d. or CV of office SBP. Table 4 shows the results of the Cox regression analyses (with or without adjustments for age, sex, BMI, habitual drinking, dipstick proteinuria (threshold $\geqslant 1 \mathrm{gl}^{-1}$ ) and mean heart rate) for composite renal end points. HRs were standardized by calculating them for 1-s.d. change in any given variables, which allow direct comparison of the prognostic value of different BP parameters. Even after adjustments for confounders were made, the s.d. of office SBP/DBP and the CV of office SBP/DBP were associated with composite renal end points. Similarly, delta in office SBP/DBP was also associated with composite renal end points. On the other hand, after adjustment, the mean office SBP/DBP and maximum office SBP/DBP were not associated with composite renal end points.

\section{Cutoff values for VVV of BP as an indicator of composite renal end point}

Supplementary Figure S1 shows receiver operating characteristic curves for s.d. of office SBP and CV of office SBP as indicators of the composite renal end point. The area under the receiver operating characteristic curve was 0.80 for s.d. of office SBP and 0.78 for CV of office SBP. The cutoff value corresponding to the maximum Youden index ((sensitivity + specificity $)-1)$ for s.d. of office SBP was $14.8 \mathrm{~mm} \mathrm{Hg}$ (sensitivity $86 \%$, specificity $63 \%$ ). The cutoff value corresponding to the maximum Youden index for CV of office SBP was $11.5 \%$ (sensitivity $76 \%$, specificity $77 \%$ ). 

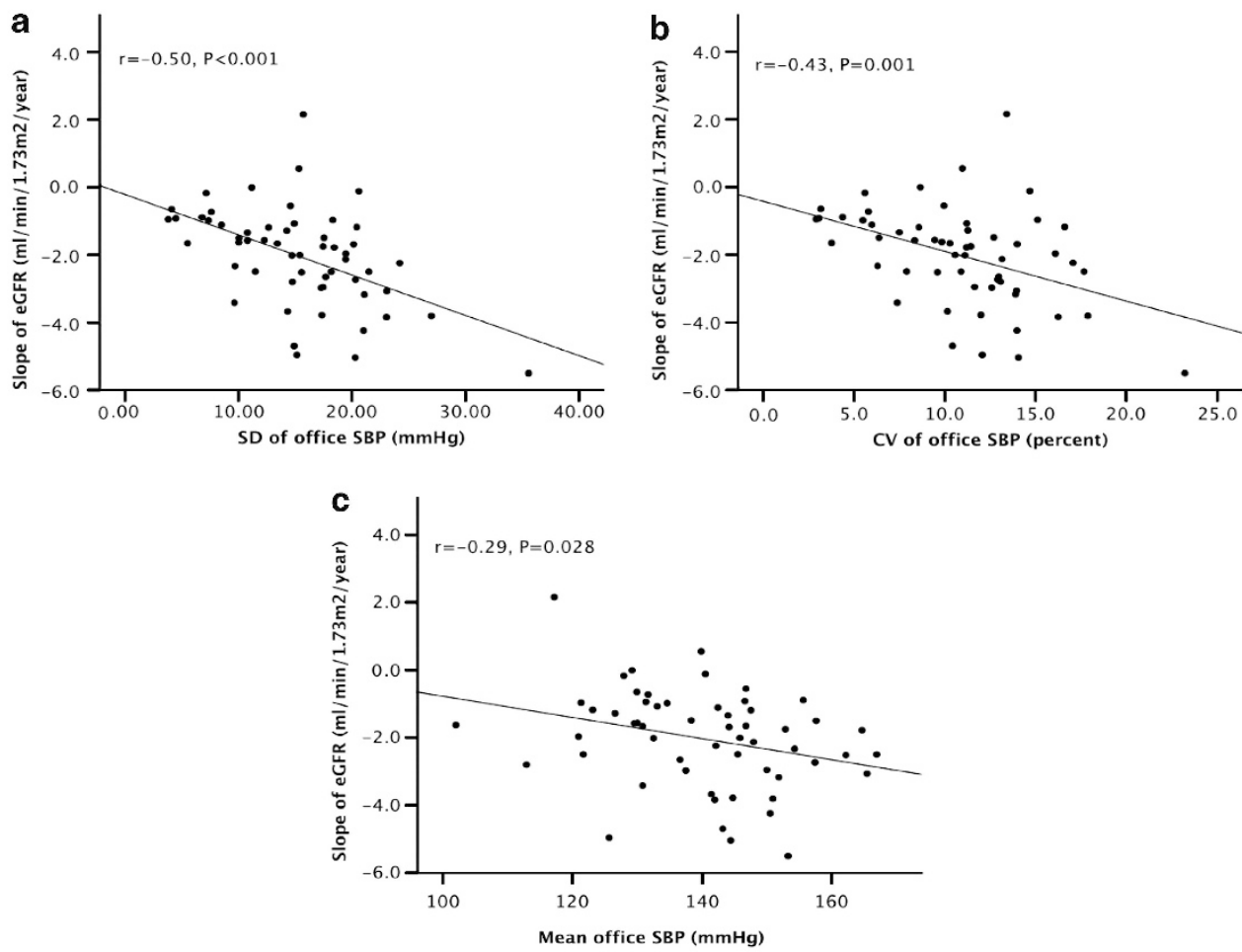

Figure 1 Simple correlations between the slope of the eGFR and s.d. of office SBP (a), CV of office SBP (b) and mean office SBP (c) in patients with non-diabetic CKD $(n=56)$. CKD, chronic kidney disease; CV, coefficient of variation; eGFR, estimated glomerular filtration rate; SBP, systolic blood pressure.

Table 3 Multivariate regression analyses between BP parameters and the slope of the eGFR in patients with non-diabetic CKD $(n=56)$

\begin{tabular}{|c|c|c|c|c|c|c|}
\hline \multirow[b]{3}{*}{ Independent variable } & \multicolumn{3}{|c|}{ Model 1} & \multicolumn{3}{|c|}{ Model 2} \\
\hline & & & Model & & & Model \\
\hline & $\beta$ & $P$ & $\mathrm{R}^{2}$ & $\beta$ & $P$ & $\mathrm{R}^{2}$ \\
\hline Mean office SBP, mm Hga & -0.24 & 0.08 & 0.13 & -0.19 & 0.23 & 0.26 \\
\hline Mean office DBP, $\mathrm{mm} \mathrm{Hg}^{\mathrm{a}}$ & -0.07 & 0.68 & 0.08 & 0.17 & 0.30 & 0.26 \\
\hline The s.d. of office SBP, $\mathrm{mm} \mathrm{Hg}^{\mathrm{b}}$ & -0.50 & $<0.001$ & 0.30 & -0.56 & $<0.001$ & 0.52 \\
\hline The s.d. of office DBP, $\mathrm{mm} \mathrm{Hg}{ }^{b}$ & -0.30 & 0.029 & 0.17 & -0.27 & 0.06 & 0.33 \\
\hline CV of office SBP, \% ${ }^{b}$ & -0.47 & 0.001 & 0.29 & -0.54 & $<0.001$ & 0.52 \\
\hline CV of office DBP, \% ${ }^{b}$ & -0.35 & 0.009 & 0.21 & -0.35 & 0.015 & 0.37 \\
\hline Maximum office SBP, $\mathrm{mm} \mathrm{Hg}{ }^{b}$ & -0.66 & 0.002 & 0.26 & -0.84 & $<0.001$ & 0.51 \\
\hline Maximum office DBP, mm Hgb & 0.11 & 0.50 & 0.10 & 0.16 & 0.39 & 0.28 \\
\hline Delta in office SBP, $\mathrm{mm} \mathrm{Hg}^{\mathrm{b}}$ & -0.51 & $<0.001$ & 0.30 & -0.57 & $<0.001$ & 0.51 \\
\hline Delta in office $\mathrm{DBP}, \mathrm{mm} \mathrm{Hg}^{\mathrm{b}}$ & -0.23 & 0.09 & 0.14 & -0.21 & 0.15 & 0.30 \\
\hline
\end{tabular}

Abbreviations: $\mathrm{BP}$, blood pressure; CKD, chronic kidney disease; CV, coefficient of variation; DBP, diastolic blood pressure; eGFR, estimated glomerular filtration rate; LDL, low-density lipoprotein; $\beta$, standardized regression coefficient; $R^{2}$, multiple coefficient of determination; SBP, systolic blood pressure.

aModel 1: These models were adjusted by age, sex, body mass index, habitual drinking, dipstick proteinuria (threshold $\geqslant 1 \mathrm{gl}^{-1}$ ) and mean heart rate. Model 2: These models were adjusted by age, sex, body mass index, habitual drinking, current smoking, statin use, fasting glucose, LDL cholesterol, dipstick proteinuria (threshold $\geqslant 1 \mathrm{gl}^{-1}$ ), and mean heart rate and mean interval between visits.

bModel 1: These models were adjusted by mean SBP, age, sex, body mass index, habitual drinking, dipstick proteinuria (threshold $\geqslant 1 \mathrm{gl}^{-1}$ ) and mean heart rate. Model 2: These models were adjusted by mean SBP, age, sex, body mass index, habitual drinking, current smoking, statin use, fasting glucose, LDL cholesterol, dipstick proteinuria (threshold $\geqslant 1 \mathrm{gl}^{-1}$ ), mean heart rate and mean interval between visits.
Differences in VVV of SBP or slope of the eGFR with respect to antihypertensive medication used

Supplementary Tables S1 and S2 show differences in the VVV of SBP with respect to antihypertensive medication used. Two groups subdivided by antihypertensive medication used (calcium channel blocker, diuretic, beta blocker and alpha blocker) were compared. There was no significant difference in the s.d. or CV of office SBP with respect to antihypertensive medication used. Supplementary Table S3 shows differences in the slope of the eGFR according to antihypertensive medication used. Use of calcium channel blockers was associated with a steeper decline in eGFR in the present study. Use of other classes of antihypertensive medication was not associated with significant difference in the slope of the eGFR.

\section{DISCUSSION}

In the present study, we demonstrated that the VVV of BP is significantly correlated with the slope of the eGFR in patients with non-diabetic CKD. In addition, our results suggested that the VVV of $\mathrm{BP}$ is significantly associated with the composite renal end point defined as a doubling of serum creatinine or the need for dialysis. To best of our knowledge, this is the first study to report a longitudinal effect of the VVV of BP in the progression of non-diabetic CKD.

Our study revealed an association between the VVV of BP and a deterioration of renal function. In a cross-sectional study by Kawai et $a l .^{20}$, it was reported that the VVV of BP correlated with albuminuria and renal vascular resistance. A study by Kilpatrick et al. ${ }^{21}$ reported that in type 1 diabetes mellitus, the VVV of BP was associated with the development of nephropathy. Although in these 

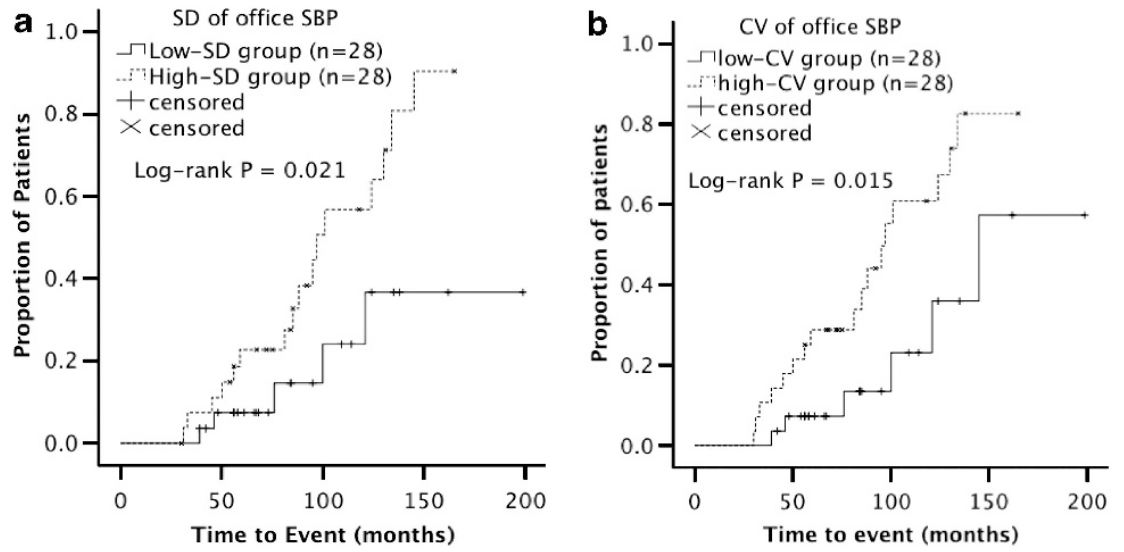

Figure 2 Kaplan-Meier curves for composite renal end point in patients with non-diabetic CKD according to the s.d. of office SBP (a) and CV of office SBP (b). The composite renal end point was defined as a doubling of serum creatinine or the need for dialysis. CKD, chronic kidney disease; CV, coefficient of variation; SBP, systolic blood pressure.

Table 4 Cox proportional hazard model: standardized HR $(95 \% \mathrm{Cl})$ for composite renal outcome associated with increase in BP parameters in patients with non-diabetic CKD $(n=56)$

\begin{tabular}{lllll}
\hline BP parameters & $H R(95 \%$ Cl) & $P$ & Adjusted HR (95\% Cl) & $P$ \\
\hline Mean office SBP & $1.00(0.63-1.59)$ & 0.99 & $0.95(0.59-1.54)$ & 0.95 \\
Mean office DBP & $0.78(0.47-1.28)$ & 0.32 & $0.86(0.47-1.58)$ & 0.86 \\
The s.d. of office SBP & $2.20(1.37-3.53)$ & 0.001 & $2.50(1.45-4.32)$ & 0.001 \\
The s.d. of office DBP & $1.70(1.07-2.57)$ & 0.023 & $2.02(1.18-3.45)$ & 0.011 \\
CV of office SBP & $2.12(1.32-3.43)$ & 0.002 & $2.36(1.35-4.13)$ & 0.003 \\
CV of office DBP & $1.96(1.20-3.19)$ & 0.007 & $2.16(1.22-3.82)$ & 0.008 \\
Maximum office SBP & $1.52(0.64-2.47)$ & 0.09 & $1.55(0.88-2.73)$ & 0.13 \\
Maximum office DBP & $0.98(0.60-1.59)$ & 0.93 & $1.19(0.60-2.38)$ & 0.62 \\
Delta in office SBP & $2.12(1.33-3.38)$ & 0.002 & $2.42(1.39-4.21)$ & 0.002 \\
Delta in office DBP & $1.63(1.06-2.53)$ & 0.027 & $1.90(1.12-3.21)$ & 0.017 \\
\hline
\end{tabular}

Abbreviations: BMI, body mass index; BUN, blood urea nitrogen; $\mathrm{Cl}$, confidence internal; CKD, chronic kidney disease; CV, coefficient of variation; HR, hazard ratio; SBP, systolic blood pressure.

Standardized HR is a change in risk for each s.d. change in variable explored. Adjustments were applied for the confounding factors of age, sex, body mass index, habitual drinking, dipstick proteinuria (threshold $\geqslant 1 \mathrm{~g}^{-1}$ ) and mean heart rate. Composite renal endpoint included doubling of serum creatinine and need for dialysis. Composite renal end point was defined as a doubling of serum creatinine or the need for dialysis.

studies, the decline in renal function was not evaluated, our data are consistent with their findings regarding the impact on renal disease of the VVV of BP. Moreover, the VVV of BP was demonstrated to be significantly associated with carotid intima-media thickness and arterial stiffness ${ }^{29}$ and endothelial dysfunction, ${ }^{40}$ independent of mean BP. Recently, endothelial dysfunction and carotid arterial thickening were reported to occur in parallel with a decline in eGFR in patients with CKD. ${ }^{41}$ The myogenic response of glomerular afferent arterioles protects the glomerulus from the damaging effects of hypertension by sensing increases in SBP and responding with compensatory vasoconstriction. ${ }^{42,43}$ Endothelial dysfunction leads to a decrease in nitric oxide ${ }^{44}$ and an increase in reactive oxygen species. ${ }^{45}$ These changes are associated with a thickening of afferent arterioles and increased preglomerular resistance, which impair glomerular autoregulation, ${ }^{45}$ make glomeruli vulnerable to hypertensive injury and deteriorate renal function. ${ }^{46}$ On the basis of these findings, it is possible to speculate that endothelial dysfunction and arterial remodeling of afferent arterioles have important roles in the impact of VVV of BP on the deterioration of renal function.
Very recently, Okada et al. ${ }^{47}$ reported that day-by-day BP variability assessed by home BP measurements had no significant association with the progression of CKD. However, their study included a wide variety of patients, including patients with conditions such as chronic glomerulonephritis and diabetic nephropathy, who were not included in the present study. The impact of BP variability may be different in these patients. In the study by Okada et al., the average observation period was shorter than in the present study (36 vs. 89 months). The observation period may not have been long enough to capture a significant difference in the decline in eGFR. Day-by-day BP variability reflects medium-term fluctuations, whereas the VVV of BP reflects long-term fluctuations. The impact on renal function may be different between medium-term BP fluctuations and long-term BP fluctuations.

There remains the question of whether the VVV of BP exerts an influence on renal function or if renal function exerts an influence on the VVV of BP. In the present study, baseline eGFR was not significantly associated with the VVV of BP (data not shown). Likewise, in the cross-sectional study by Kawai et al. ${ }^{20}$, eGFR was not significantly associated with the VVV of BP. Considering the temporal relationship between the VVV of BP and deterioration of renal function observed in the present study, it is reasonable to assume that the VVV of BP does influence renal function.

In the present univariate analysis, mean office SBP was associated with the slope of the eGFR; however, the association was no longer significant after adjustment for possible confounding factors. In addition, the increase in mean office SBP was not associated with a significantly increased risk of composite renal end points. Many studies have suggested that mean SBP is an important risk factor for deterioration of renal function in patients with CKD. ${ }^{6-11}$ However, these studies included a wide variety of patients such as those with diabetes mellitus or nephritis. A few studies have investigated the impact of mean SBP on the deterioration of renal function solely in patients with non-diabetic CKD. Consistent with the present study, the results of a multivariate analysis by Strojceva-Taneva et al. ${ }^{48}$ demonstrated that in 70 patients with non-diabetic CKD, mean SBP was not a significant variable. In the present study, the absence of a significant association between mean SBP and the deterioration of renal function may have been due to a type 2 error due to the small sample size; however, the present results did reveal a stronger impact of the VVV of BP than mean SBP on the deterioration of renal function. 
In the present study, maximum office SBP was significantly associated with the slope of the eGFR, independent of the mean office SBP, but maximum office SBP was not a significant variable according to the Cox regression model analysis of renal composite endpoint. We have recently shown that maximum home SBP was significantly associated with urinary albumin excretion after adjustment for confounders, but maximum home SBP did not provide useful information about potential kidney damage associated with the mean home SBP level. ${ }^{49}$ Accumulating evidence suggests that urinary albumin excretion is an early sign of damage in the vasculature of the kidney. ${ }^{50}$ These findings may indicate that although maximum office SBP is associated with renal damage, the association may not be as strong as that between the VVV of SBP and renal damage. Kidneys may be more vulnerable to long-term SBP variability (VVV of BP) than episodic increases in SBP (maximum office SBP).

In the present study, the delta in office SBP was associated with the slope of the eGFR, independent of the mean office SBP, and was a significant variable in the Cox regression model of renal composite end point. We have demonstrated that the delta in office SBP was associated with carotid intima-media thickness, ${ }^{29}$ which has been reported to be associated with an increased risk of CKD. ${ }^{41,51}$ Wide oscillations in BP are considered to increase the extent of oscillatory shear stress in the endothelium, initiating atherosclerosis formation by the stimulation of adhesion molecule expression. ${ }^{52}$ It is possible to speculate that oscillatory shear stress in the endothelium of glomerular afferent arterioles stimulates atherosclerosis formation and impairs glomerular autoregulation, ${ }^{45}$ which leads to renal dysfunction. ${ }^{46}$

In the present study, there was no significant difference in the s.d. of office SBP according to the class of antihypertensive medication administered. This finding seems inconsistent with those of recent reports showing that calcium channel blockers lowered the VVV of $\mathrm{SBP}^{53,54}$ or short-term BP variability, as assessed by ambulatory blood pressure monitoring. ${ }^{55,56}$ A possible explanation for this discrepancy is that the sample size of the present study may have been too small to detect different effects on the VVV of BP according to the class of antihypertensive medication. Use of calcium channel blockers was associated with a steeper decline in eGFR in the present study. One possible explanation for this association is that patients with a long history of hypertension (who may have impaired glomerular autoregulation and be vulnerable to the VVV of $\mathrm{BP}$ ) tended to be prescribed calcium channel blockers. It has been demonstrated that calcium channel blockers are the most frequently prescribed agents for the treatment of hypertension in Japan (in patients receiving monotherapy, 69\%; in the patients receiving combination therapy, $89.6 \%) .{ }^{57}$ In the present study, the number of patients administered other classes of antihypertensive medication was small, which may account for why the association with the slope of the eGFR was not significant for these other classes.

\section{Study limitations}

The present study has several limitations, including its small sample size and its retrospective nature. A prospective study with a larger population will be needed to further clarify associations between the VVV of BP and progression of non-diabetic CKD. Another limitation is that the albumin excretion rate was not included. However, dipstick proteinuria (threshold $\geqslant 1 \mathrm{gl}^{-1}$ ) was recently reported to be a stronger predictor of rapid renal function decline than albuminuria. ${ }^{28}$ In the present study, we adjusted for the presence of dipstick proteinuria (threshold $\geqslant 1 \mathrm{gl}^{-1}$ ) in the Cox hazard model. Moreover, the intervals between visits varied widely in this study.
However, the reproducibility of the VVV of BP has been reported recently in a study indicating that the first seven and second seven SBP measurements identified similar groups of individuals with higher BP variability. ${ }^{58}$ This finding may suggest that the impact of VVV of BP on the deterioration of renal function can be reproduced, even if different intervals between visits are used. Treatment effects on the VVV of BP during the observation period should also be mentioned in this context. We were unable to demonstrate a significant difference in the s.d. of office SBP according to classes of antihypertensive agent. In addition, in patients with treated hypertension (Anglo-Scandinavian Cardiac Outcomes Trial Blood Pressure Lowering Arm), the residual VVV of SBP on treatment was shown to be a strong predictor of stroke and coronary events, irrespective of the class of antihypertensive drug. ${ }^{18}$

\section{Perspectives}

The clinical implications of the present study include the idea that the VVV of SBP may be useful as a predictor of worsening renal function in patients with non-diabetic CKD, based on the cutoff values we proposed herein. A higher VVV of SBP in patients with non-diabetic $\mathrm{CKD}$ can herald poorer renal outcome, which requires a more careful attention during the follow-up. However, little is known about the utility of the VVV of SBP as a therapeutic target. It is possible to consider that the VVV of SBP could serve as a therapeutic target. Intervention studies of means of improving the VVV of SBP will be required to evaluate this hypothesis.

\section{CONFLICT OF INTEREST}

The authors declare no conflict of interest.

\section{ACKNOWLEDGEMENTS}

We would like to thank a doctor (SY) for her assistance with the study. There is no previous presentation of the whole or part of the work presented in the article.

1 Coresh J, Selvin E, Stevens LA, Manzi J, Kusek JW, Eggers P, Van Lente F, Levey AS. Prevalence of chronic kidney disease in the United States. JAMA 2007 298: 2038-2047

2 Gilbertson DT, Liu J, Xue JL, Louis TA, Solid CA, Ebben JP, Collins AJ. Projecting the number of patients with end-stage renal disease in the United States to the year 2015 J Am Soc Nephrol 2005; 16: 3736-3741.

3 Stengel B, Billon S, Van Dijk PC, Jager KJ, Dekker FW, Simpson K, Briggs JD. Trends in the incidence of renal replacement therapy for end-stage renal disease in Europe, 1990-1999. Nephrol Dial Transplant 2003; 18: 1824-1833.

4 Rifkin DE, Shlipak MG, Katz R, Fried LF, Siscovick D, Chonchol M, Newman AB, Sarnak MJ. Rapid kidney function decline and mortality risk in older adults. Arch Intern Med 2008; 168: 2212-2218.

5 Al-Aly Z, Zeringue A, Fu J, Rauchman MI, McDonald JR, El-Achkar TM, Balasubramanian S, Nurutdinova D, Xian $\mathrm{H}$, Stroupe $\mathrm{K}$, Abbott KC, Eisen S. Rate of kidney function decline associates with mortality. J Am Soc Nephrol 2010; 21: 1961-1969.

6 Shulman NB, Ford CE, Hall WD, Blaufox MD, Simon D, Langford HG, Schneider KA Prognostic value of serum creatinine and effect of treatment of hypertension on renal function. Results from the hypertension detection and follow-up program. The Hypertension Detection and Follow-up Program Cooperative Group. Hypertension 1989; 13: 80-93.

7 Walker WG, Neaton JD, Cutler JA, Neuwirth R, Cohen JD. Renal function change in hypertensive members of the Multiple Risk Factor Intervention Trial. Racial and treatment effects. The MRFIT Research Group. JAMA 1992; 268: 3085-3091.

8 de Goeij MC, Voormolen N, Halbesma N, de Jager DJ, Boeschoten EW, Sijpkens YW, Dekker FW, Grootendorst DC. The PREPARE-1 study group. Association of blood pressure with decline in renal function and time until the start of renal replacement therapy in pre-dialysis patients: a cohort study. BMC Nephrol 2011; 12: 38-47.

9 Brazy PC, Stead WW, Fitzwilliam JF. Progression of renal insufficiency: role of blood pressure. Kidney Int 1989; 35: 670-674.

10 Jovanovic DB, Djukanovic L. Analysis of factors influencing chronic renal failure progression. Ren Fail 1999; 21: 177-187.

11 Locatelli F, Marcelli D, Comelli M, Alberti D, Graziani G, Buccianti G, Redaelli B, Giangrande A. Proteinuria and blood pressure as causal components of progression to 
end-stage renal failure. Northern Italian Cooperative Study Group. Nephrol Dial Transplant 1996; 11: 461-467.

12 Whaley-Connell AT, Sowers JR, Stevens LA, McFarlane SI, Shlipak MG, Norris KC, Chen SC, Qiu Y, Wang C, Li S, Vassalotti JA, Collins AJ, Kidney Early Evaluation Program Investigators. CKD in the United States: Kidney Early Evaluation Program (KEEP) and National Health and Nutrition Examination Survey (NHANES) 19992004. Am J Kidney Dis 2008; 51 (4 Suppl 2): S13-S20.

13 Chobanian AV, Bakris GL, Black HR, Cushman WC, Green LA, Izzo Jr JL, Jones DW, Materson BJ, Oparil S, Wright Jr JT, Roccella EJ, National Heart, Lung, and Blood Institute Joint National Committee on Prevention, Detection, Evaluation, and Treatment of High Blood Pressure, National High Blood Pressure Education Program Coordinating Committee. The Seventh Report of the Joint National Committee on Prevention, Detection, Evaluation, and Treatment of High Blood Pressure: the JNC 7 report. JAMA 2003; 289: 2560-2572.

14 Kidney Disease Outcomes Quality Initiative (K/DOQI). K/DOQI clinical practice guidelines on hypertension and antihypertensive agents in chronic kidney disease. $\mathrm{Am} J$ Kidney Dis 2004; 43(5 Suppl 1): S1-290.

15 Rosendorff $\mathrm{C}$. Hypertension and coronary artery disease: a summary of the American Heart Association scientific statement. J Clin Hypertens 2007; 9: 790-795.

16 Hata Y, Kimura Y, Muratani H, Fukiyama K, Kawano Y, Ashida T, Yokouchi M, Imai $\mathrm{Y}$, Ozawa T, Fujii J, Omae T. Office blood pressure variability as a predictor of brain infarction in elderly hypertensive patients. Hypertens Res 2000; 23: 553-560.

17 Hata Y, Muratani H, Kimura Y, Fukiyama K, Kawano Y, Ashida T, Yokouchi M, Imai Y, Ozawa T, Fujii J, Omae T. Office blood pressure variability as a predictor of acute myocardial infarction in elderly patients receiving antihypertensive therapy. J Hum Hypertens 2002; 16: 141-146.

18 Rothwell PM, Howard SC, Dolan E, O'Brien E, Dobson JE, Dahlöf B, Sever PS, Poulter NR. Prognostic significance of visit-to-visit variability, maximum systolic blood pressure, and episodic hypertension. Lancet 2010; 375: 895-905.

19 Muntner P, Shimbo D, Tonelli M, Reynolds K, Arnett DK, Oparil S. The relationship between visit-to-visit variability in systolic blood pressure and all-cause mortality in the general population: findings from NHANES III, 1988 to 1994. Hypertension 2011; 57: 160-166.

20 Kawai T, Ohishi M, Kamide K, Onishi M, Takeya Y, Tatara Y, Oguro R, Yamamoto K, Sugimoto K, Rakugi $H$. The impact of visit-to-visit variability in blood pressure on renal function. Hypertens Res 2012; 35: 239-243.

21 Kilpatrick ES, Rigby AS, Atkin SL. The role of blood pressure variability in the development of nephropathy in type 1 diabetes. Diabetes Care 2010; 33: 2442-2447.

22 Bakris GL, Sarafidis PA, Weir MR, Dahlof B, Pitt B, Jamerson K, Velazquez E, StaikosByme L, Kelly RY, Shi V, Chiang YT, Weber MA. Renal outcomes with different fixeddose combination therapies in patients with hypertension at high risk for cardiovascular events (ACCOMPLISH): a prespecified secondary analysis of a randomized controlled trial. Lancet 2010; 375: 1173-1181.

23 National Kidney Foundation. K/DOQI clinical practice guidelines for chronic kidney disease: evaluation, classification, and stratification. Am J Kidney Dis 2002; 39: S1-266.

24 Hollenberg NK, Raij L. Angiotensin-converting enzyme inhibition and renal protection. An assessment of implications for therapy. Arch Intern Med 1993; 153: 2426-2435.

25 The fifth report of the Joint National Committee on Detection, Evaluation, and Treatment of High Blood Pressure (JNC V). Arch Intern Med 1993; 153: 154-183.

26 The sixth report of the Joint National Committee on prevention, detection, evaluation, and treatment of high blood pressure. Arch Intern Med 1997; 157: 2413-2446.

27 Rosman JB, ter Wee PM, Meijer S, Piers-Becht TP, Sluiter WJ, Donker AJ. Prospective randomised trial of early dietary protein restriction in chronic renal failure. Lancet 1984; 2: 1291-1296.

28 Clark WF, Macnab JJ, Sontrop JM, Jain AK, Moist L, Salvadori M, Suri R, Garg AX. Dipstick proteinuria as a screening strategy to identify rapid renal decline. J Am Soc Nephrol 2011; 22: 1729-1736.

29 Nagai M, Hoshide S, Ishikawa J, Shimada K, Kario K. Visit-to-visit blood pressure variations: new independent determinants for carotid artery measures in the elderly at high risk of cardiovascular disease. J Am Soc Hypertens 2011; 5: 184-192.

30 Matsuo S, Imai E, Horio M, Yasuda Y, Tomita K, Nitta K, Yamagata K, Tomino Y, Yokoyama H, Hishida A. Revised equations for estimated GFR from serum creatinine in Japan. Am J Kidney Dis 2009; 53: 982-992.

31 Brenner BM, Cooper ME, de Zeeuw D, Keane WF, Mitch WE, Parving HH, Remuzzi G, Snapinn SM, Zhang Z, Shahinfar S. RENAAL Study Investigators. Effects of losartan on renal and cardiovascular outcomes in patients with type 2 diabetes and nephropathy. N Engl J Med 2001; 345: 861-869.

32 Ohira T, Tanigawa T, Tabata M, Imano H, Kitamura A, Kiyama M, Sato S, Okamura T, Cui R, Koike KA, Shimamoto T, Iso H. Effects of habitual alcohol intake on ambulatory blood pressure, heart rates, and its variability among Japanese men. Hypertension 2009; 53: 13-19.
33 Cahan A, Ben-Dov IZ, Bursztyn M. Association of heart rate with blood pressure variability: implications for blood pressure measurement. Am J Hypertens 2012; 25: 313-318.

34 Nagasawa Y, Yamamoto R, Rakugi H, Isaka Y. Cigarette smoking and chronic kidney diseases. Hypertens Res 2012; 35: 261-265.

35 Kalaitzidis RG, Elisaf MS. The role of statins in chronic kidney disease. Am J Nephrol 2011; 34: 195-202.

36 Fox CS, Larson MG, Leip EP, Meigs JB, Wilson PW, Levy D. Glycemic status and development of kidney disease: the Framingham Heart Study. Diabetes Care 2005; 28: 2436-2440.

37 Oda E, Kawai R. Low-density lipoprotein (LDL) cholesterol is cross-sectionally associated with preclinical chronic kidney disease (CKD) in Japanese men. Intern Med 2010; 49: 713-719.

38 Youden SWJ. Index for rating diagnostic tests. Cancer 1950; 3: 32-35.

39 Lindeman RD, Tobin J, Shock NW. Longitudinal studies on the rate of decline in renal function with age. J Am Geriatr Soc 1985; 33: 278-285.

40 Diaz KM, Veerabhadrappa P, Kashem MA, Feairheller DL, Strugeon KM, Williamson ST, Crabbe DL, Brown MD. Relationship of visit-to-visit and ambulatory blood pressure variability to vascular function in African Americans. Hypertens Res 2012; 35: 55-61.

41 Yilmaz MI, Stenvinkel P, Sonmez A, Saglam M, Yaman H, Kilic S, Eyileten T, Caglar K, Oguz Y, Vural A, Cakar M, Altun B, Yenicesu M, Carrero JJ. Vascular health, systemic inflammation and progressive reduction in kidney function; clinical determinants and impact on cardiovascular outcomes. Nephrol Dial Transplant 2011; 26: 3537-3543.

42 Chen J, Sgouralis I, Moore LC, Layton HE, Layton AT. A mathematical model of the myogenic response to systolic pressure in the afferent arteriole. Am J Physiol Renal Physiol 2011; 300: F669-F681.

43 Loutzenhiser R, Bidani A, Chilton L. Renal myogenic response: kinetic attributes and physiological role. Circ Res 2002; 90: 1316-1324.

44 Tousoulis D, Kampoli AM, Tentolouris C, Papageorgiou N, Stefanadis C. The role of nitric oxide on endothelial function. Curr Vasc Pharmacol 2012; 10: 4-18.

45 Carlström M, Lai EY, Ma Z, Steege A, Patzak A, Eriksson UJ, Lundberg JO, Wilcox CS, Persson AE. Superoxide dismutase 1 limits renal microvascular remodeling and attenuates arteriole and blood pressure responses to angiotensin 2 via modulation of nitric oxide bioavailability. Hypertension 2010; 56: 907-913.

46 Loutzenhiser R, Griffin KA, Bidani AK. Systolic blood pressure as the trigger for the renal myogenic response: protective or autoregulatory? Curr Opin Nephrol Hypertens 2006; 15: 41-49.

47 Okada T, Matsumoto H, Nagaoka Y, Nakao T. Association of home blood pressure variability with progression of chronic kidney disease. Blood Press Monit 2012; 17: $1-7$

48 Strojceva-Taneva O, Selim G, Stojkovski L, Ivanovski N. Hypertension and progression of nephropathy in diabetic and non-diabetic chronic kidney disease patients. Hippokratia 2007; 11: 72-76.

49 Matsui Y, Ishikawa J, Eguchi K, Shibasaki S, Shimada K, Kario K. Maximum value of home blood pressure: a novel indicator of target organ damage in hypertension. Hypertension 2011; 57: 1087-1093.

50 de Jong PE, Gansevoort RT. Albuminuria in non-primary renal disease: risk marker rather than risk factor. Nephrol Dial Transplant 2010; 25: 656-658.

51 Chonchol M, Gnahn H, Sander D. Impact of subclinical carotid atherosclerosis on incident chronic kidney disease in the elderly. Nephrol Dial Transplant 2008; 23: 2593-2598.

52 Chappell DC, Varner SE, Nerem RM, Medford RM, Alexander RW. Oscillatory shear stress stimulates adhesion molecule expression in cultured human endothelium. Circ Res 1998; 82: 532-539.

53 Webb AJ, Rothwell PM. Effect of dose and combination of antihypertensives on interindividual blood pressure variability: a systematic review. Stroke 2011; 42 . 2860-2865.

54 Webb AJ, Fischer Y, Mehta Z, Rothwell PM. Effects of antihypertensive-drug class on interindividual variation in blood pressure and risk of stroke: a systematic review and meta-analysis. Lancet 2010; 375: 906-915.

55 Ichihara A, Kaneshiro Y, Takemitsu T, Sakoda M. Effects of amlodipine and valsartan on vascular damage and ambulatory blood pressure in untreated hypertensive patients. $J$ Hum Hypertens 2006; 20: 787-794.

56 Zhang Y, Agnoletti D, Safar ME, Blacher J. Effect of antihypertensive agents on blood pressure variability: the Natrilix SR versus candesartan and amlodipine in the reduction of systolic blood pressure in hypertensive patients (X-CELLENT) study. Hypertension 2011; 58: 155-160.

57 Mori H, Ukai H, Yamamoto H, Saitou S, Hirao K, Yamauchi M, Umemura S. Current status of antihypertensive prescription and associated blood pressure control in Japan. Hypertens Res 2006; 29: 143-151.

58 Muntner P, Joyce C, Levitan EB, Holt E, Shimbo D, Webber LS, Oparil S, Re R, Krousel-Wood M. Reproducibility of visit-to-visit variability of blood pressure measured as part of routine clinical care. J Hypertens 2011; 29: 2332-2338.

Supplementary Information accompanies the paper on Hypertension Research website (http://www.nature.com/hr) 